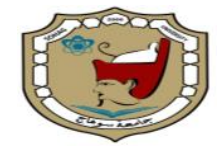

Sohag University

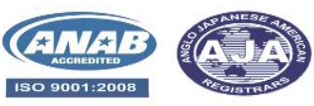

Sohag Medical Journal

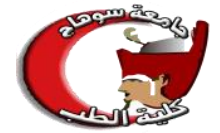

Faculty of Medicine

\title{
A New Synthetic Cannabinoid Substance, AB-CHMINACA: Review Article
}

\author{
Rasha Elhaddad Ali Mousa ${ }^{1}$, Sahar Mohamed Gebril ${ }^{2}$, Khaled Masoud \\ Mohamed Masoud ${ }^{3}$, Rania Ahmad Radwan ${ }^{4}$, Soheir Ali Mohammad ${ }^{4}$. \\ 1-Department of Forensic Medicine and Clinical Toxicology, Faculty of Medicine, Sohag \\ University, Egypt. \\ 2- Department of Histology, Faculty of Medicine, Sohag University, Egypt \\ 3-Department of Forensic Chemistry, Naif Arab University for Security Sciences, \\ Riyadh, Saudi Arabi. \\ 4-Department of Forensic Medicine and Clinical Toxicology, Faculty of Medicine, \\ Sohag University, Egypt.
}

\begin{abstract}
The synthetically new illicit drugs which are called New psychoactive substances (NPS) are the calamity of the modern era. Their danger is much more than the natural drugs of abuse and at the same time, they cannot be detected on regular drug screens making diagnosis very difficult. The number of NPS is growing very fast making their detection more complicated. Another challenge is that their health effects are not well studied and cannot be predicted. Synthetic cannabinoids (SCs) are the most prevalent group in all available NPS. One recent member of the SCs group is AB-CHMINACA. This review article summarizes the available data about AB-CHMINACA. The obtained data were summarized under the following subtitles; historical background, chemical structure, classification, physical characters, pharmacokinetics and pharmacodynamics, toxicity, methods of detection, the magnitude of the problem, and the situation in Egypt. The reviewed studies reveal that AB-CHMINACA like other SC substances are considered toxic with high liability for dependence. Most of the available studies are case reports. The available literature is lacking in specific organ pathology and well-structured toxicity studies.
\end{abstract}

Keywords: AB-CHMINACA, Cannabinoids, NPS

\section{Introduction}

New psychoactive substances (NPS) are considered a major health threat and a medico-legal challenge all over the world. Although they are gaining the atention of health workers and police authorities, still the information about their $\mathrm{h}$ ealth hazards is insufficient. Another challenge is the speed at which new substances appear making researchers take

the responsibility of evaluating the risk and detecting new substances. This helps authorities banning their trading and consumption ${ }^{[1]}$. Classification of NPS is $b$ ed on their effects compared to the old drugs of abuse. They include Synthetic cannabinoids, Synthetic Cathinones, Synthetic opioids, benzodiazepine analogs, and many others. ${ }^{[2]}$ 
Synthetic cannabinoids (SCs) are substances that are created in the lab to give the same psychoactive action as tetrahydrocannabinol (THC), the main active substance in Cannabis. Cannabis is the most widely used substance of abuse worldwide, which is legally banned in most countries. SCs may have chemical structures related to THC, but most of them have different structures making them undetectable by usual screening $\mathrm{m}$ ethods. ${ }^{[3]}$ They emerged in the drug market as first, second, and most recent third-generation substances. ${ }^{[4]}$

\section{Material and methods}

Searching the available literature for all types of articles: review articles, observational studies, and experimental studies. The used search engines were google scholar and Egyptian Knowledge Bank. The used keywords: AB-CHMINACA, synthetic cannabinoids, THC, and Strix in Egypt. The revised data were sumarized and arranged under the following subtitles: historical background, chemical structure, classification, physical characters, pharmacodynamics and pharmacokinetics, toxicity, methods of detection, the magnitude of the problem, and the situation in Egypt.

\section{Historical background}

The plant cannabis Sativa is the most popular plant for recreational purposes for many centuries. There are many active substances isolated from this plant, the major one is tetrahydrocannabinol (THC). ${ }^{[3]}$ Cannabis is banned in most countries including Egypt. However, a small number of countries still have legal use of cannabis as medicine for example Canada, Netherlands, Spain, Israel, and some states in the United States. The only legally prescribed cannabinoid medications are Nabilone, Dronabinol, and THC-cannabidiol mixture. They are pre- scribed for selected cases as those with severe vomiting associated with anticancer chemotherapy, severe chronic pain, spasticity, and control marijuana withdrawal symptoms. However, these medications still have abuse potential that makes their use very limited [5and6] Many researchers tried to synthesize substances that act on the cannabinoid receptors without the side effects of $\triangle 9$-THC. The beginning was in the late 1970s and early 1980s. ${ }^{[7]}$ The results of the research showed that these substances have adverse effects, and they never found their way to a commercial drug. ${ }^{[8]}$

In the early 2000s, new illicit substances emerged in the form of herbal mixtures. A few years later chemical analysis showed it was a mixture of new SC substances. These substances were added to schedule I for drug control this stimulated the factories of the illicit drug market to create other new substances going around in a circle of banning and synthesizing new drugs. [9]

In 2009, the UNODC stated that SCs are the most reported NPS in the illicit drugs market. The number of SC substances that were reported in 2009 was 31 . The number increased to reach 154 in 2013. As a result of the rapid emergence of new substances, synthetic cannabinoids are still the most reported substance in the NPS group. ${ }^{[10]}$

AB-CHMINACA is a new SC substance. It was reported by Pfizer as a trial for a commercial drug, but the project failed due to the development of side effectts. ${ }^{[11]}$ In 2013, AB-CHEMINACA was first detected in ER cases of acute intoxication as a new illicit drug. ${ }^{[12]}$

\section{Chemical structure and Classification}

Chemical structure is shown in Figure (1). AB-CHMINACA is an abbreviation 
for its chemical name " $\mathrm{N}-[(2 \mathrm{~S})-1$-amino3-methyl-1-oxo-2-butanyl]-1-

(cyclohexyl-methyl) -1H- indazole-3-carboxamide"[13].

According to structural relation to THC, AB-CHMINACA is an SC that has a chemical structure not related to THC. So, it is considered non-classical in its chemical structure. ${ }^{[3]}$ classification according to its relation to the SC family, AB-CHMINACA is an Indazole carboxamide derivative of the group Naphthylmethylindoles. ${ }^{[13]}$ Regarding its emergence in the illicit drug market, AB-CHMINACA is a third-generation SC. $[4,14]$

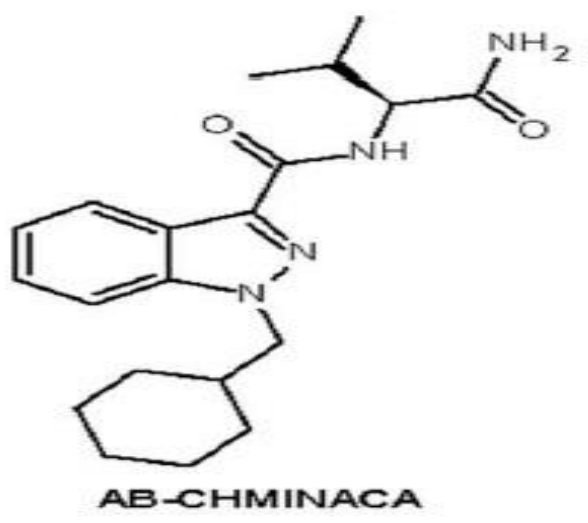

Figure (1): chemical structure of ABCHMINACA ${ }^{[13]}$.

\section{Physical characters}

Available preparations: AB-CHMINAC$A$ and other cannabinoids are prepared in the lab as crystal or powder substances. The substance is dissolved in organic solvent then the solution is sprayed over other herbs that may be inactive substances or upon cannabis itself to give stronger effects. The herbs and the substance upon it are usually smoked to give effects like cannabis. Packages are commonly labeled as herbal incense, not for human use Figures (2), this is the commonest form. ${ }^{[3]}$ Another form is the cliquid for an electronic cigarette. ${ }^{[9]}$ Also, the pure form itself is sold as it is and reported cases that had been found to have the substance in their stomach suggesting oral administration.[15] Oth-er rare preparations are soaked paper and letters with the substance that was reported as a drug-smuggling method for prisoners in the UK. ${ }^{[16]}$

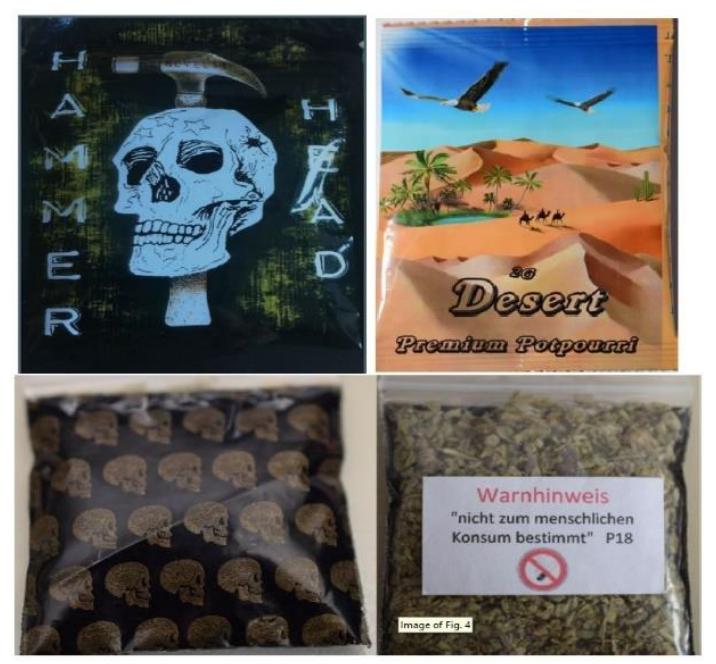

Figure (2): Different packages of SC labelled as not for human consumption [36].

\section{Pharmacokinetics}

The most common route of administration is inhalation via water pipe, bong, cigarette, or blunt. Other routes have been also recorded as vaporized administeration, oral and even rectal administration. ${ }^{[17]}$

Theoretically, the effect can be obtained as small as $0.5-1 \mathrm{mg}$ if smoked. The dose is larger if ingested due to decreased oral bioavailability. ${ }^{[18]}$ However, the real consumption dose is highly variable as there is no precise amount is applied to each package and the amount received peruse cannot be predicted. The absorption across alveolar membranes is assumed to be very rapid that the onset of action begins within few minutes. ${ }^{[19]}$

AB-CHMINACA is rapidly distributed to different body organs as Pancreas, ad- 
ipose tissue, kidney, liver, heart muscles, brain, lung, and spleen. It has a high affinity to adipose tissue.[20] AB-CHMINACA also can be detected in the hair of abusers. [21and22]

AB-CHMINACA is rapidly metabolized. ${ }^{[23]}$ In vitro and in vivo studies showed 26 metabolites of AB CHMINACA shown in Figure.(3) The main metabolic reaction is hydroxylation by cytochrome p450. Another pathway is carboxylation by amidase and glucuronidation. ${ }^{[14]}$
The parent drug is lipophilic, so it is present to a lower extent in the urine. Most metabolites of AB-CHMINACA are detected in urine.[14]

Although studies are limited, there is evidence of obstetric complications and fetal affection on the use of synthetic cannabinoids during pregnancy. Eclampsia, craniofacial anomalies, and withdrawal symptoms in the newborn were reported. [24and 25]

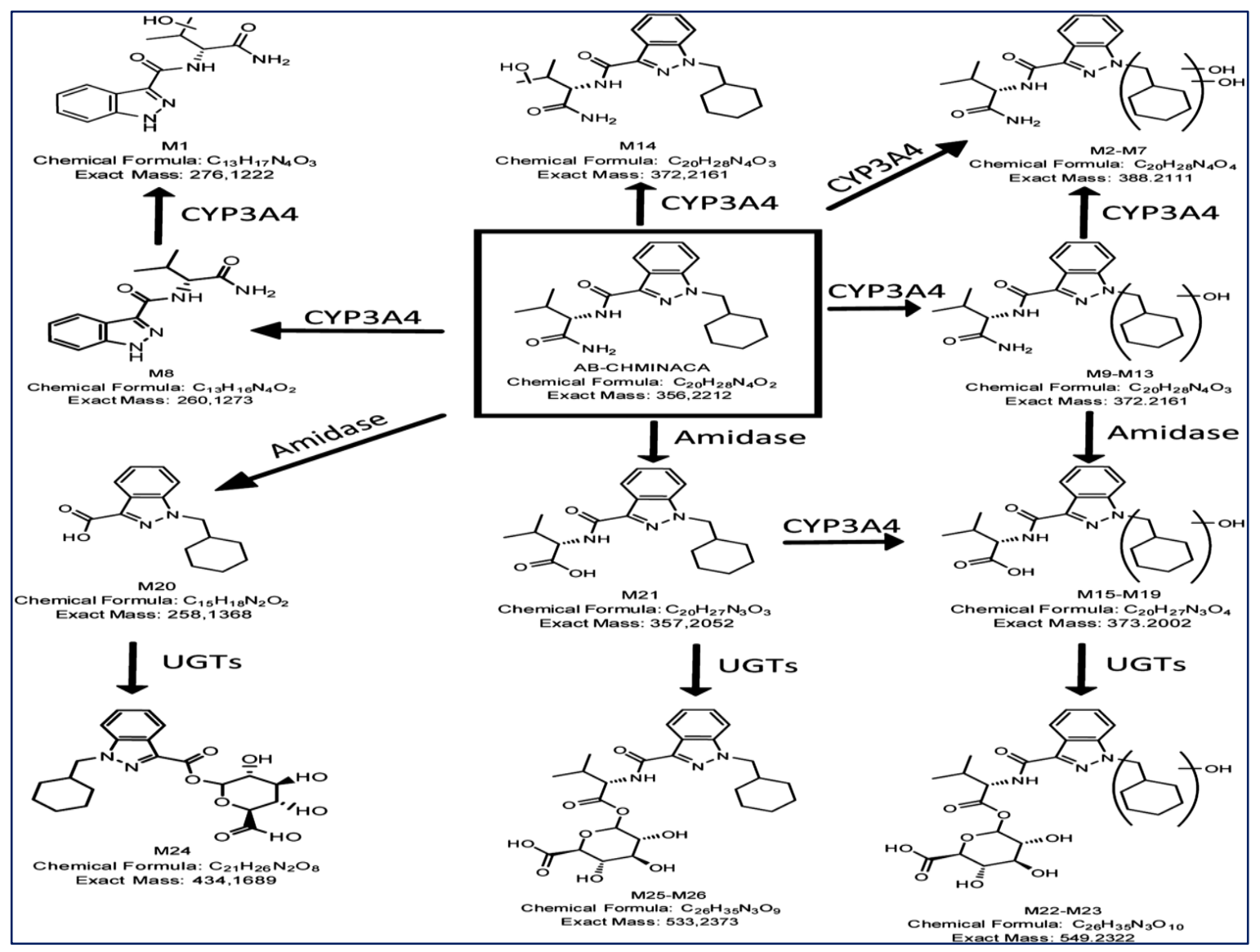

Figure (3): Metabolic pathways of AB-CHMINACA ${ }^{[15]}$.

\section{Pharmacodynamics}

AB-CHMINACA acts through the ordinary cannabinoid receptors, $\mathrm{CB} 1$ and $\mathrm{CB}$ 2. $\mathrm{CB} 1$ receptors are primarily found in the central and peripheral nervous systems. CB1 is also found at a lower extent in other tissues as immune cells, testes, heart, and blood vessels. On the other hand, $\mathrm{CB} 2$ receptors are present outside the nervous system, mainly in cells of the immune system and to a lesser extent 
in hematopoietic cells and CNS.[7] However, other receptors are involved but to a lesser degree. ${ }^{[3]}$ These other receptors are responsible for the nonCB1/CB2 actions of cannabinoids. However, the minor actions by other receptors are not evoked by all types of cannabinoids .[26]

Although THC and other SCRAs all are acting through the same receptors, there are differences in their actions due to the presence of multiple ligands and pathways of intracellular signaling on stimulation of cannabinoid receptors ${ }^{[27]}$ T-his is a promising research area, still a window to develop an SCRA that has desired medical action without the undesired side effects ${ }^{[28]}$.

\section{Potency}

In general, SCs are far more potent than THC. THC is considered a partial agonist while most SCs are full agonists. ${ }^{[29]} \mathrm{In}$ vitro, AB-CHMINACA was tested for binding to CB1 receptors, and it was found to be a full agonist. Also, it was found that $\mathrm{AB}-\mathrm{CHMINACA}$ has potency 11 to 58 times more than THC in the different components of the mouse tetrad test. ${ }^{[30]}$

\section{Pharmacological actions and toxicity}

Acting on the same receptors, SCs have actions like THC. CB1 receptors are responsible for the anti-nociceptive effects that control neuropathic pain and the psychotropic effects of THC as well. While $\mathrm{CB} 2$ receptors are responsible for immune response mediated by THC. ${ }^{[31 a n d}$ 32].

The clinical picture of acute toxicity of AB-CHMINACA is like other SCs. Many studies and case reports are available describing the acute toxic effects observed in ER patients. The most common presentation is neuropsychiatric abnormalities and the most serious is cardiop- ulmonary complications. Also, GIT manifestations are very common. Other system affection includes acute kidney injury and acute liver failure. Clinical presentation includes excitation, depression, agitation, psychosis, convulsions, hyperemesis, hypotension or hypertension, infarction, stroke, respiratory depression or tachypnea, lung edema, visual disturbance, suicidal behavior, and even death from direct toxicity to body organs ${ }^{[9 a n d 33]}$ The toxic dose in humans is unkno-wn. Even animal studies are inadequate regarding toxicity. There is no available data about the LD50 of ABCHMI-NACA. However, LD50 of THC and other cannabinoids suggesting low leth-ality.

There is no specific treatment is known till now, only symptomatic, and supportive measures according to the case or treatment of associated consumption. ${ }^{[15]}$

Regarding the abuse potential, no studies are evaluating the potential liability of AB-CHMINACA to produce dependence and withdrawal symptoms on discontinuation of the drug. Studies on other synthetic cannabinoid substances proved the development of withdrawal symptoms in abusers mainly anxiety and tachycardia. Administration of THC did not relieve the symptoms probably due to adulteration or co-ingestion. There are no specific measures to control the withdrawal symptoms, but they can be calmed using benzodiazepines. ${ }^{[34]}$

\section{Manner of death and intoxication}

Most cases are accidental overdoses in patients with a history of drug abuse. ${ }^{[35]}$ Less frequent are suicidal cases wi-th associated depression or other problems. ${ }^{[15]}$

\section{Methods of detection}

Because of different chemical structures, Synthetic cannabinoids cannot be cross- 
reacting with THC detection kits. Chromatography techniques are preferred for the identification and detection of many drugs.[36] AB-CHMINACA and its $\mathrm{m}$ etabolites were successfully detected in urine, blood, hair, and different tissue samples using Gas-chromatography mass spectrometry (GC-MS), high-performance liquid chromatography (HPLC), liquid chromatography time of flight mass spectrometry (LC-TOFS).[37and38]

\section{Magnitude of the problem}

It is difficult to estimate the total consumption of synthetic cannabinoids all over the world because not all countries have a good surveillance system. However, some countries have annual reports on the consumption of drugs of abuse. As mentioned by United Nations Office on Drug and Crime (UNODC), from data collected from 29 countries, the crosssectional analysis represents the problem at interval time between 2016-2018, half of the reported new psychoactive substance (NPS) was synthetic cannabinoids and opioids together while Synthetic cannabinoids (Synthetic cannabinoid receptor agonists or SCRAs) were a little bit higher than opioids. ${ }^{[39]}$

\section{The situation in Egypt}

As a part of the global problem, Egypt suffers the same growth rate of NPS consumption. There is no national surveillance to estimate the actual total number of NPS abusers. However, there are few separate epidemiologic studies on different population groups indicating that the problem is present and growing up.

Hashim et al., made surveillance in five different faculties at Cairo University to evaluate the prevalence of NPS among students. Their study showed that three fourth of abusers are male students, $6.8 \%$ consumed strox and $0.5 \%$ consum- ed voodoo. Also, there was a strong relationship between tobacco and hashish smoking and strong usage. ${ }^{[40]}$

In another study, the emergency cases admitted to Ain-Shams University hospital, a poison control center were assessed for SC exposure in the interval of $18 \mathrm{mo}-$ nths from the beginning of the year 2018. The total number of cases was more than five hundred patients and $96 \%$ of them were males. Strix consumption was the most prevalent, $70 \%$ of cases, followed by voodoo, then other substances. ${ }^{[41]}$

Regarding legislation, drugs with abuse potential are categorized into five schedules. These schedules are arranged from I to $\mathrm{V}$ according to the abuse potential of the contained drugs. Schedule I drugs have the highest liability of abuse while schedule V drugs have the least. Also, drugs of schedule I have no medical role and their trade is illegal. However, drugs in schedules from II to $\mathrm{V}$ have medical uses that can be legal in certain situations. In Egypt, AB-CHMINACA was added to schedule I controlled substances by the Controlled Substance Act 440, 2018. ${ }^{[42]}$

\section{References}

1. Elliott, S. and Evans, J. A 3-year review of new psychoactive substances in casework. Forensic Science International. 2014; 243: 55-60.

2. Zawilska, J. B. and Wojcieszak, J. Novel psychoactive substances: Classification and general information. In Synthetic cathinones: Novel addictive and stimulatory psychoactive substances, Zawilska, J. B. Cham, Springer International Publishing: 2018, pp 11-24. Part of the Current Topics in Neurotoxicity book series

3. Hudson, S. and Ramsey, J. The emergence and analysis of synthetic cannabinoids. Drug Testing and Analysis. 2011; 3(7-8): 466-478. 
4. Kukura-Hanajiri, R.; Kawamura, N. U. M., and Goda, Y. Changes in the prevalence of new psychoactive substances before and after the introduction of the generic scheduling of synthetic cannabinoids in Japan. Drug testing and analysis. 2014; 6(7-8): 832839.

5. Haney, M.; Cooper, Z. D.; Bedi, G.; Vosburg, S. K.; Comer, S. D. and Foltin, R. W. Nabilone Decreases Marijuana Withdrawal and a Laboratory Measure of Marijuana Relapse. Neuropsychopharmacology. 2013; 38(8): 1557-1565.

6. Tsang, C. C. and Giudice, M. G. Nabilone for the Management of Pain. Pharmacotherapy: The Journal of Human Pharmacology and Drug Therapy. 2016; 36(3): 273-286.

7. Banister, S., D.; Moir, M.; Stuart, J.; Kevin, R., C.; Wood, K., E.; Longworth, M.; et al., Pharmacology of Indole and Indazole Synthetic Cannabinoid Designer Drugs AB-FUBINACA, ADBFUBINACA, AB-PINACA, ADBPINACA, 5F-AB-PINACA, 5FADB-PINACA, ADBICA, and 5FADBICA. American Chemical society chemical neuroscience. 2015; 6(9):15461559.

8. Guindon, J. and Hohmann, A. G. Cannabinoid cb2 receptors: A therapeutic target for the treatment of inflammatory and neuropathic pain. British Journal of Pharmacology. 2008; 153(2): 319-334.

9. Hermanns-Clausen, M.; Müller, D.; Kithinji, J.; Angerer, V.; Franz, F.; Eyer, F.; et al., Acute side effects after consumption of the new synthetic cannabinoids AB-CHMINACA and MDMB-CHIMICA. Clinical Toxicology. 2018; 56(6): 404-411.

10.UNODC. world drug report 2015.

11.Buchler, I.; Hayes, M.; Hedge, S.; Ackerman, S.; Jones, D.; Kortum, S.; et al, Indazole Derivatives as CB1 Receptor Modulators and Their Preparation and Use in the Treatment of CB1-Mediated Diseases. 2009; Patent
WO 2009/106982, New York, NY: Pfizer Inc.

12.Uchiyama, N.; Shimokawa, Y.; Kawamura, M.; Kikura-Hanajiri, R. and Hakamatsuka, T. Chemical analysis of a benzofuran derivative, 2-(2ethylaminopropyl) benzofuran (2EAPB), eight synthetic cannabinoids, five cathinone derivatives, and five other designer drugs newly detected in illegal products. Forensic Toxicology. 2014; 32(2): 266-281.

13.Kądzioła-Długołęcka，K.; Frączak, O.; Tkacz-Szczęsna, B.; Kijewska, I.; Wilczek, R.; et al., The paths of syntheses, chemical characteristics and stability tests for selected synthetic cannabinoids: 5F-PB-22, NM-2201, UR144, and AB-CHMINACA. Australian Journal of Forensic Sciences. 2019; 1-12.

14.Erratic, C.; Negreira, N.; Norouzizadeh, H.; Covaci, A.; Neels, H.; Maidens, K. et al., In vitro and in vivo human metabolism of the synthetic cannabinoid AB-CHMINACA. Drug Testing and Analysis. 2015; 7(10): 866-876.

15.Klaus, J.; Gorenjak, M. and Marinsek, M. Suicide attempt with a mix of synthetic cannabinoids and synthetic cathinones: A case report of non-fatal intoxication with AB-CHMINACA, AB-FUBINACA, alpha-PHP, alpha-PVP, and 4-CMC. Forensic Science International. 2016; 265: 121-124.

16.Ford, L. T., and Berg J. D. Analytical evidence to show letters impregnated with novel psychoactive substances are a means of getting drugs to inmates within the UK prison service. Annals of Clinical Biochemistry. 2018; 55(6): 673-678.

17.Vandrey, R.; Dunn, K. E.; Fry, J. A. and Girling, E. R. A survey study to characterize use of Spice products (synthetic cannabinoids). Drug and Alcohol Dependence. 2012; 120(1-3): 238-241.

18.WHO. AB-CHMINACA, Review Report. Expert Committee on Drug Dependence, Thirty-ninth Meeting. 2017; Agenda item 4.1. 
19.Peterson, B. L. and Couper, F. J. Concentrations of AB-CHMINACA and AB-PINACA and Driving Behavior in Suspected Impaired Driving Cases. Journal of analytical toxicology. 2015; 39(8): 642-647.

20.Hasegawa, K.; Kurita, A.; Minakata, K.; Gonmori, K.; Nozawa, H.; Yamagishi, I.; et al., Postmortem distribution of $\mathrm{AB}$ CHMINACA, 5-fluoro-AMB, and piperidine in body fluids and solid tissues in a fatal poisoning case: usefulness of adipose tissue for detection of the drugs in unchanged forms. Forensic Toxicology. 2014; 33(1): 45-53.

21.Franz, F.; Angerer, V.; HermannsClausen, M.; Auwärter, V. and Moosmann, B. Metabolites of synthetic cannabinoids in hair-proof of consumption or false friends for interpretation? Analytical and bioanalytical chemistry. 2016; 408(13): 3445-3452.

22.Sim, J.; Cho, H. S.; Lee, J.; In, S. and Kim, E. Determination of ab-chminaca and its metabolites in human hair and their deposition in the hair of abusers. Journal of Pharmaceutical and Biomedical Analysis. 2017; 140: 162168.

23.Maeda, H.; Kikura-Hanajiri, R.; Kawamura, M.; Nagashima, E. and Yoshida, K.I. AB-CHMINACA-induced sudden death from non-cardiogenic pulmonary edema. Clinical Toxicology. 2018; 56(2): 143-145.

24.Gilbert, M. T.; Sulik, K. K.; Fish, E. W.; Baker, L. K.; Dehart, D. B. and Parnell, S. E. Dose-dependent teratogenicity of the synthetic cannabinoid CP-55,940 in mice. Neurotoxicology and teratology. 2016; 58: 15-22.

25.Orsolini, L.; Papanti, D.; Corkery, J.; De Luca, M. A.; Cadoni, C.; Di Chiara, G. et al., Is there a teratogenicity risk associated with cannabis and synthetic cannabimimetics'('spice') intake? CNS $\&$ Neurological Disorders-Drug Targets. 2017; 16(5): 585-591.
26.Brown, A. J. Novel cannabinoid receptors. British Journal of Pharmacology. 2007; 152(5): 567-575.

27.Patel, M.; Manning, J. J.; Finlay, D. B.; Javitch, J. A.; Banister, S. D.; Grimsey, N. L. et al., Signalling profiles of a structurally diverse panel of synthetic cannabinoid receptor agonists. Biochemical Pharmacology. 2020; 175: 113871.

28.Wouters, E.; Walraed, J.; Banister, S. D. and Stove, C. P. Insights into biased signaling at cannabinoid receptors: synthetic cannabinoid receptor agonists. Biochemical Pharmacology. 2019; 169: 113623.

29.Castagneto, M. S.; Gorelick, D. A.; Desrosiers, N. A.; Hartman, R. L.; Pirard, S. and Huestis, M. A. Synthetic cannabinoids: epidemiology, pharmacodynamics, and clinical implications. Drug and alcohol dependence. 2014; 144: 12-41.

30.Wiley, J. L.; Marusich, J. A.; Lefever, T. W.; Antonazzo, K. R.; Wallgren M. T.; Cortes, R. A.; et al., AB-CHMINACA, AB-PINACA, and FUBIMINA: affinity and potency of novel synthetic cannabinoids in producing $\Delta 9$ Tetrahydrocannabinol-like effects in mice. The Journal of Pharmacology and Experimental Therapeutics. 2015; 354:28-339.

31.Buckley, N. E.; McCoy, K. L.; Mezey, É.; Bonner, T.; Zimmer, A.; Felder, C. C.; et al., Immunomodulation by cannabinoids is absent in mice deficient for the cannabinoid CB2 receptor. European journal of pharmacology. 2000; 396(2-3): 141-149.

32.Ziring, D.; Wei, B.; Velazquez, P.; Schrage, M.; Buckley, N. E. and Braun, $\mathrm{J}$. Formation of $\mathrm{B}$ and $\mathrm{T}$ cell subsets require the cannabinoid receptor $\mathrm{CB} 2$. Immunogenetics. 2006; 58: 714-725.

33.Solimini, R.; Busardo, F. P.; Rotolo, M.C.; Ricci, S.; Mastrobattista, L.; Mortali, C.; et al., Hepatotoxicity associated to synthetic cannabinoids use. European Review for Medical and 
Pharmacological Sciences. 2017; 21(1): 1:6.

34.Nacca, N.; Vatti, D.; Sullivan, R.; Sud, P.; Su, M. and Marraffa, J. The synthetic cannabinoid withdrawal syndrome. Journal of Addiction Medicine. 2013; 7(4): 296-298.

35.Angerer, V.; Jacobi, S.; Franz, F.; Auwarter, V. and Pietsch, J. Three fatalities associated with the synthetic cannabinoids 5F-ADB, 5F-PB-22, and AB-CHMINACA. Forensic Science International. 2017; 281: e9-e15.

36.Namera, A.; Kawamura, M.; Nakamoto, A.; Saito, T. and Nagao, $M$. Comprehensive review of the detection methods for synthetic cannabinoids and cathinones. Forensic Toxicology. 2015; 33(2): 175-194.

37.Logan, B. K.; Reinhold, L. E.; Xu, A. and Diamond, F. X. Identification of synthetic cannabinoids in herbal incense blends in the united states. Journal of forensic sciences. 2012; 57(5): 11681180.

38.Tsujikawa, K.; Yamamuro, T.; Kuwayama, K.; Kanamori, T.; Iwata, Y. $\mathrm{T}$. and Inoue, $\mathrm{H}$. Thermal degradation of a new synthetic cannabinoid QUPIC during analysis by gas chromatographymass spectrometry. Forensic Toxicology. 2014; 32(2): 201-207.
39.UNODC. Current NPS Threats. Volume 1. 2019.

40.Hashim, A. M.; Hassan, A. M.; El-Din Amin, G. E. and Allam, M. F. Prevalence of strox smoking among university students in cairo, egypt. The Open Public Health Journal. 2020; 13(1).

41.Hashem, A.; Mahmoud, S.; Abou Anza, R. and Abdel Hamid, W. Pattern of acute synthetic cannabinoids toxicity in patients presented to the poison control center of ain shams university hospitals. Ain Shams Journal of Forensic Medicine and Clinical Toxicology. 2021; 36(1): 112.

42.Ministry of Health and Population, act number. 440 for the year 2018. The following substances, and, their isomers, their esters, their ethersand salts of their isomers are added to the second sectionof schedule 1 of the act number 128 for the year 1960: AB-Fubinaca, AB-Chminaca, XLR-11, XLR-11N-4(fluoropentyl) isomer, FUB-AMB and 5 flouro ADB The official gazette of Egypt. 2018; 199:3-4 